\section{The second RSSA short course on Current Ethical Issues in Radiology: 31 July 2010}

Dr Clive Sperryn

Following the success of the first short course in Cape Town in January, it was repeated in the Investec Auditorium in Sandton. The workshop was well attended, with 210 delegates. The four topics (as in the January course) were:

- Ethical Considerations in Cochlear Implant Patients, presented by Dr Derrick Wagenfeld

- Self-referral - A Moral Hazard, by Dr Clive Sperryn

- Paediatric Imaging: The Ethics of Radiation Exposure, by Professor Savvas Andronikou

- Patient Confidentiality in Clinical Practice: Is HIV Status Still Relevant? by Professor Keymanthri Moodley

- Kick-backs and Perverse Incentives, by Dr Bates Alheit. This included a discussion of cases and actions taken by the RSSA in such instances, by Esme Prins who also highlighted the new rules published by the HPCSA. These are accessible via the HPCSA website, which is included as a link on the RSSA website. CPD ethics points were updated and very positive feedback received. Many attendees experienced the convenience of the Gautrain, and the venue will certainly be useful for future meetings in the area. Congratulations to Professor Leon Janse van Rensburg, who again arranged an excellent course, and thanks to the ConsultUS team for the organising, to Covidien for sponsorship, and to Investec for use of the auditorium and generous catering.

\section{The First RSSA/SGR Gastrointestinal Radiology Course: Stellenbosch, 7 - 9 August 2010}

Professor Steve Beningfield

This first combined meeting between the RSSA and the Society of Gastrointestinal Radiologists International Education Conference, held at the Spier Estate just outside Stellenbosch, was a thoroughly wellorganised and enjoyable gathering, despite some chilly showers which doubled the flow of the adjacent Eerste River overnight.

Proceedings were conducted under the guidance of some of the foremost international GI radiology experts - Jay Heiken, Michael Federle, Sat Somers (who originally hails from Durban), Kumar Sandrasegaran, Christine Menias, (Egyptian-born), Erik Paulson, Jonathan Kruskal (ex-UCT, recently appointed Chair at Beth Israel Deaconess Medical Centre) and Larry Schwartz, who presented an update on the RECIST (Response Evaluation Criteria In Solid Tumours) criteria.

Imaging in all the key abdominal regions, including the hollow muscular organs and solid viscera, was comprehensively covered in a refresher/update format, well-delivered for both the starters and experienced attendees. A wide range of pathological topics was covered, including blunt and penetrating trauma, benign disease and malignancy, inflammatory and infective entities, and surgical and iatrogenic issues.

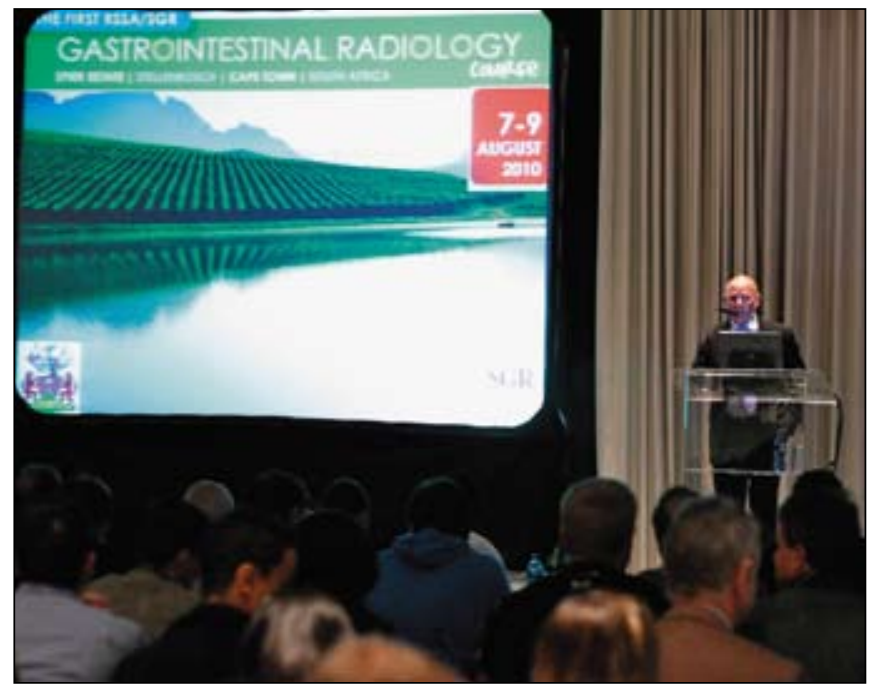

Welcome address by Professor Leon I van Rensburg, RSSA Congress Chair.

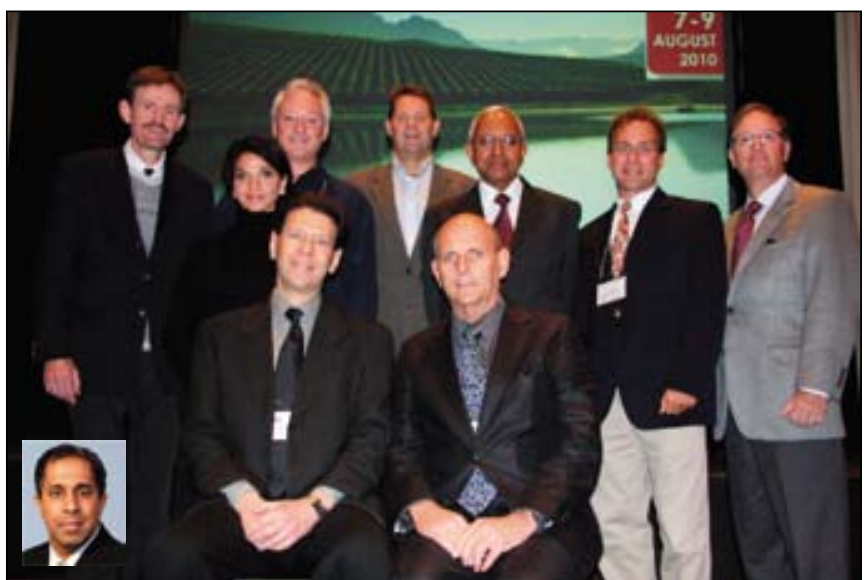

The faculty, standing from left to right: Professor Steve Benningfield (UCT, Scientific Committee Chair), Dr Christine Menias (USA), Professor Jonathan Kruskal (USA), Professor Erik Paulsen (USA), Professor Sat Somers (Canada), Dr Clive Sperryn (RSSA president), Professor Mickael Federle (USA), Sitting: Professor lay P Heiken (SGR president) and Professor Leon J van Rensburg (RSSA Congress Chair). Inset: Dr Kumar Sandrasegaran (USA).

An excellent overview of the embryological basis of peritoneal anatomy clarified many of the confusing aspects of this complex area. Logical walk-throughs of duplex Doppler and CT techniques preceded some of the more detailed presentations.

The techniques of grading splenic and liver injuries, and the preeminent role of conservative management, coupled with interventive radiology to deal with arterial injury, bile leaks and drainages, came through clearly. The value of the sentinel clot sign providing reliable evidence indicating the origin of the bleed (by virtue of the higher density clot in that area, versus the lesser density elsewhere) and haematocrit-sign of anticoagulation, were convincingly illustrated by case material.

The role of imaging before and after liver transplantation was also presented, including split liver transplants and the attendant complexities, both surgical and radiological. Liver masses, both benign and malignant, received attention, with the increasing value 
of hepatocyte-specific MRI agents in focal nodular hyperplasia; increasing accumulation of this contrast implies biliary excretion and hence functional hepatocytes. Disappointingly, well-differentiated hepatocellular carcinomas can behave in a similar fashion.

A riveting presentation by Jonathan Kruskal, including in vivo video images of liver tumour deposition and angiogenesis in a rat model, highlighted some innovative research on the processes involved in tumour-seeding in the liver. In the rat, the hepatic veins are the site of tumour cell implantation. The evolution of angiogenesis in the subsequent expansion of the tumour implant was also graphically illustrated. Another thought-provoking point was the fact that DNA analysis of HCC recurrences in transplanted livers show that the cell lines are from the patient's original tumour and not from the transplanted liver, implying that the cells remain elsewhere in the body until re-manifesting in the donor liver. The technically challenging realm of liver diffusion weighted imaging to analyse tumours and their response to therapy has a number of hurdles to overcome before becoming a mainstream clinical tool.

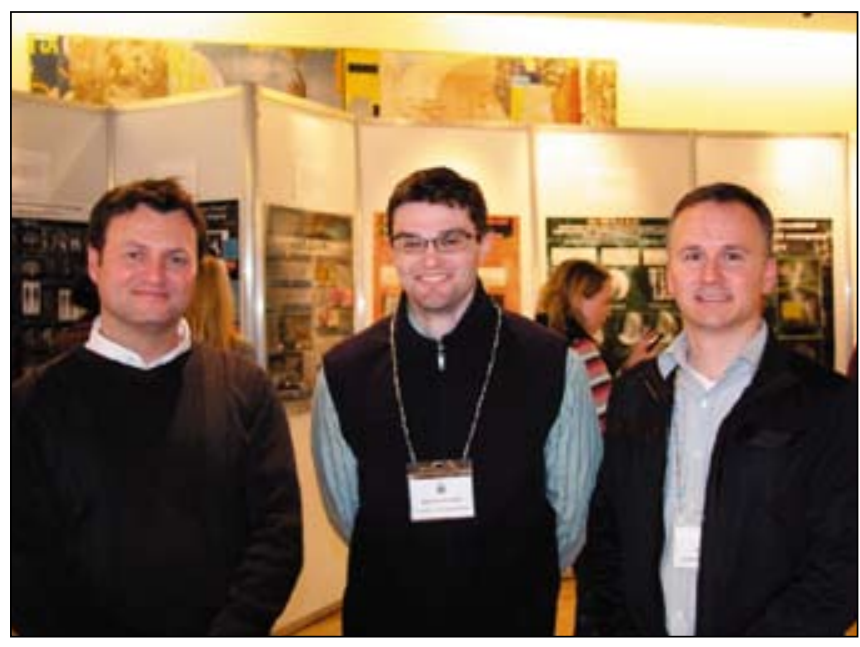

Winners of the poster competition were (from left to right) Dr Arthur Maydell (US, 3rd place), Dr Matthew Goodier(on behalf of Dr Ernst Boshoff) (Wits, 1st place) and Dr Hein Els (US, 2nd place).

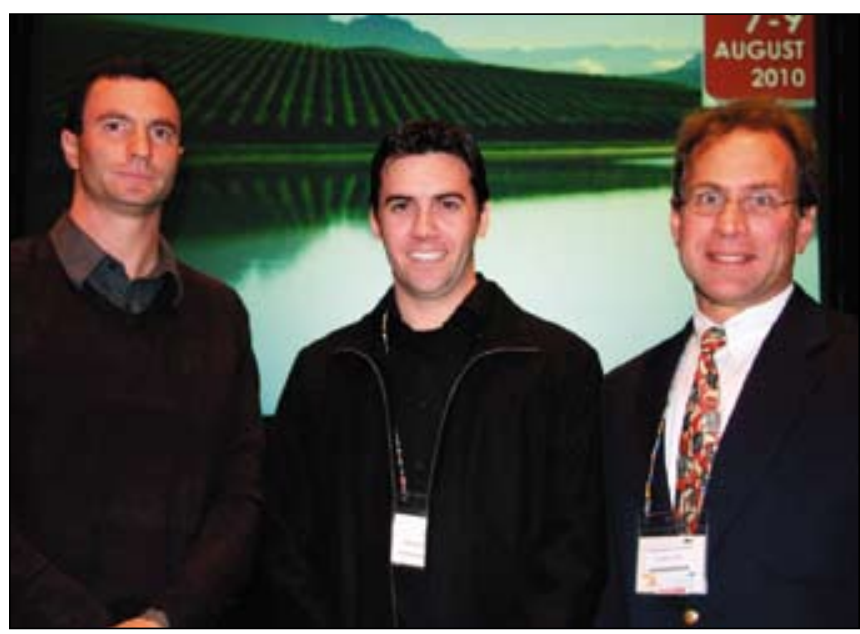

The RSSA 2010 Travel Award winners were (from left to right) Dr Andrew Lawson (UCT, RSSA Award and SGR Prize), and Dr Shaun Scheepers (US, RSSA Award), with Dr Clive Sperryn (RSSA President).
Biliary calculous disease (don't forget the pre-contrast CT), imaging and intervention in iatrogenic laparoscopic cholecystectomy biliary injuries, and the benign and malignant spectrum of biliary disease, were presented, with particular emphasis on cholangiocarcinoma in its various forms (mass forming, periductal infiltrating and intraductal growing). Also covered were some of the more important congenital biliary disorders such as Caroli's disease, as well as primary and other forms of sclerosing cholangitis.

Pancreatitis in both acute and chronic forms, with the new concept of 'peripancreatic fat necrosis; (as distinct from a pseudocyst) was detailed. The expected appearances following pancreatic surgery were also shown in detail, this including imaging following a Whipple's resection, Frey's procedure and other pancreatic drainages. The newly described entity of autoimmune pancreatitis was further clarified, reminding us to look for the loss of lobulation (giving rise to a sausage shape), a peri-pancreatic halo of tissue and other associated autoimmune conditions, with IgG4 elevation.

The subtypes of main-duct and side-branch intraductal pancreatic mucinous neoplasms (IPMNs) with differing risks of malignancy give rise to management controversies. The imaging of the viscous gelatinous secretions was convincingly demonstrated. Other cystic pancreatic lesions such as the serous and mucinous cystadenomas and the rarer solid papillary epithelial neoplasms were clarified. The major category of pancreatic adenocarcinoma was then tackled in a useful and pragmatic manner, emphasising the importance of carefully checking the portal and superior mesenteric veins (as well as the usual SMA).

A concentrated review of the art of localising pathology using 'steam-powered' barium imaging in the gut emphasised its continuing relevance, particularly given that the material was from the hallowed grounds of Harvard University. Small bowel imaging, inflammatory bowel disease, and refinements in the techniques of CT colonography all enjoyed separate presentations. Unique looks at problems with enteral lines, swallowing pathology and pelvic floor imaging and defaecography provided expert advice on less common conditions. The looming roles of endoscopic ultrasound and capsule endoscopy were flagged. The important contribution of CT in the diagnosis of appendicitis was discussed, along with the problems and potential benefits of positive and negative contrast of the bowel in CT in general. Hernia imaging by barium and CT techniques, as well as the imaging of small bowel obstruction, with particular attention being paid to closed loop configurations, was covered. The key observation here was of the tapering conjunction of two loops of bowel, indicating a twist or shared constriction.

Investigation of abdominal pain was tempered by increasing awareness of radiation risk. Trauma in its blunt and penetrating forms, abdominal haemorrhage and mesenteric ischaemia were all addressed. The puzzling entity of non-occlusive mesenteric ischaemia (NOMI) on the basis of mesenteric vasoconstriction, and the more mechanical causes of arterial or venous thrombosis, together with the less common vasculitides, were also covered.

Early detection of ruptured or threatening abdominal aortic aneursyms was mentioned by a few speakers, demystifying the highattenuating crescent and draped aorta signs, as well as the tangential 
calcification and calcium gap sign as early warnings of impending rupture

Included was an informative and well-illustrated local presentation on the local imaging of HIV and TB manifestations from Marc Mendelson and Christelle Ackerman.

'Boards' cases tested the audience's perceptions and lateral thinking, including the classic missing viscera, pointing us in the right direction e.g. no colon or testis or kidney. The number of posters from a good range of institutions was welcome, with three noteworthy oral presentations competing for the RSSA award that was finally split, with Andrew Lawson also receiving the SGR award for his presentation on self-expanding metal stents.

The turnout was excellent, particularly considering that the meeting was held over a long weekend. The scenic surroundings of Spier with the excellent catering, conference planning and support by vendors added to the value of proceedings. This was yet another tribute to the organising skills and persuasive capabilities of our Congress Chairman, Professor Leon Janse van Rensberg. Hats off to the RSSA and SGR on this initiative, and welcome to the SGR in what will hopefully become a long-term relationship leading to the strengthening of our local abdominal imaging skills.

Thanks to Dr Hein Els (Tygerberg Hospital) for the photo's.

\section{Abstracts of oral presentations at the first RSSA/SGR Gastrointestinal Radiology Course: Stellenbosch, 7 - 9 August 2010}

(presenting author first)

Imaging the Rex recessus vein pre-operatively using wedged hepatic venous portography and the role of post-operative doppler ultrasound in Rex shunts

Andrew Lawson, Groote Schuur Hospital

Paul Rischbieter, Themba Hospital

Alp Numanoglu, Nicky Wieselthaler, Red Cross War Memorial Children's Hospital

Background. In children with extrahepatic portal vein obstruction (EHPVO), formation of a mesentericoportal bypass (Rex shunt) restores hepatopedal flow, relieves portal hypertension and reduces variceal bleeding. The Rex shunt is created by inserting a vein graft between the superior mesenteric vein and the umbilical segment of the left portal vein (Rex vein). We report our experience with wedged hepatic venous portography (WHVP) in the pre-operative evaluation of the Rex vein and the post-operative ultrasound findings.

Methodology. A retrospective chart review was done in patients with EHPVO who had been considered for a mesoportal bypass between January 2001 and January 2010 at Red Cross War Memorial Children's Hospital.

Results. Sixteen patients (13 boys, 3 girls, mean age 5, range 1 - 13 years) were considered for mesoportal bypass including 4 post reduced-size liver transplant patients. Ten patients (62\%) underwent WHVP. The Rex vein was clearly identified in $8(80 \%)$ patients. A poorly canalised Rex vein was reported in one of these cases yet found to be functionally viable at surgery. One Rex vein was seen at surgery despite not being demonstrated at WHVP. Six mesoportal bypasses were performed without WHVP of which 3 (50\%) were successful. Two of the patients who underwent WHVP were post liver transplant patients; a patent Rex vein was demonstrated in both. The average shunt sizes on the first post-operative day as documented using Doppler ultrasonography was $7.8 \mathrm{~mm}$ with an average flow of 20.8 $\mathrm{cm} / \mathrm{s}$. The average flow rate increased to $27.1 \mathrm{~cm} / \mathrm{s}$ after 1 month. All shunt occlusions $(N=1)$ or partial thromboses $(N=2)$ were detected by post-operative Doppler ultrasound.

Conclusion. Our series demonstrates the use of WHVP as an effective tool with a sensitivity of $80 \%$ and specificity of $100 \%$ in the preoperative patency assessment of the Rex vein. The sensitivity of WHVP in patients who had undergone liver transplantation remains above $90 \%$. Doppler ultrasonography is useful for evaluating the size and flow rates in the post-operative shunt and is sensitive (100\%) to the detection of early shunt thrombosis.

\section{Abdominal lymphadenopathy in children with tuberculosis presenting with respiratory symptoms}

Shaun Scheepers, Savvas Andronikou, Ayanda Mapukata, Peter Donald, Stellenbosch University and Tygerberg Hospital

Background. Tuberculosis (TB) remains one of the leading causes of childhood morbidity and mortality. Pulmonary TB (PTB) is the most common form; however, extrapulmonary $\mathrm{TB}$ is on the increase, with abdominal TB being most prevalent in the paediatric age group. The radiologic hallmark of primary TB in children is lymphadenopathy. Ultrasound is a sensitive tool for detecting abdominal lymphadenopathy and may have an important role in the primary investigation of suspected TB in children.

Objectives. To determine the prevalence of abdominal lymphadenopathy in children with culture-positive PTB, presenting with respiratory symptoms; to determine whether the presence of abdominal TB could predict the presence of thoracic (hilar and mediastinal) lymphadenopathy, given the predictable course of lymphatic spread; and to define the role of ultrasound in the primary investigation of suspected TB in children.

Materials and methods. Chest radiographs and abdominal ultrasound reports of 47 children, with culture-positive PTB and respiratory symptoms, were reviewed. The prevalence of abdominal lymphadenopathy and thoracic lymphadenopathy was determined. The relative effectiveness of ultrasound for predicting thoracic lymphadenopathy was determined using $2 \times 2$ tables.

Results. The prevalence of abdominal lymphadenopathy was $19 \%$ $(N=9)$ as demonstrated on ultrasound. Thoracic lymphadenopathy was reported in $70 \%$ of our study participants. Abdominal ultrasonography had a sensitivity of $18 \%$ and a specificity of $79 \%$ for predicting thoracic lymphadenopathy when chest radiography was used as the radiological reference standard. Abdominal ultrasound did, however, identify one additional case of lymphadenopathy that was not detected on chest radiography. 


\title{
RADIOACTIVE NEWS
}

Conclusion. Since patients were selected on the basis of having respiratory TB, the fact that $19 \%$ had abdominal lymphadenopathy is noteworthy. Owing to its poor sensitivity and overall accuracy, ultrasound cannot be used in isolation for the prediction of thoracic lymphadenopathy. It is therefore suggested that ultrasound remains a valuable supplementary tool for diagnosing TB in children, due to its safety, portability and affordability.

Effectiveness of percutaneously placed self-expanding metal stents for palliation of malignant biliary obstruction

Andrew Lawson (principle investigator), S Burmeister, J Krige, Groote Schuur Hospital

P Rischbieter, Themba Hospital

Background. Percutaneously placed self-expanding metal stents (SEMS) have been widely used for palliation of malignant biliary obstruction as an alternative to major bypass surgery or when endoscopic drainage is not technically feasible. We report our experience in the placement of SEMS at Groote Schuur Hospital. Methods. A retrospective chart review was done of patients who had percutaneously placed SEMS for obstructing biliary malignancy between May 2008 and February 2010. Data included demographic information, level of biliary obstruction, efficacy and complications of SEMS insertion. Boston Scientific 6F, $69 \mathrm{~mm}^{\star} 10 \mathrm{~mm}$, Wallstent SEMS were used.

Results. Forty patients (23 men, 17 women, mean age 61, range 48 - 74 years) underwent percutaneous SEMS. Stent insertion was successful in all patients. Ten patients had obstruction at the level of the hilum, 5 in the mid common bile duct, and 25 in the low common bile duct. In 15 (37.5\%) patients, SEMS were placed during the first attempt at biliary decompression. Five (12.5\%) patients required bilateral SEMS insertion. The mean serum bilirubin decreased from $313 \mu \mathrm{mol} / \mathrm{l}$ to $127 \mu \mathrm{mol} / \mathrm{l}$ (60\% decrease) 5 days post stent insertion. Mean hospital stay post stent insertion was 5.1 days. Nine patients (22.5\%) developed complications unrelated to SEMS insertion that included gastric outlet obstruction requiring endoscopic stenting $(N=6)$, myocardial ischaemic events $(N=2)$ and pneumonia $(N=1)$. Eight patients $(20 \%)$ developed a procedure-related complication that included cholangitis after stent insertion $(N=3)$, cholangitic liver abscess $(N=1)$, subphrenic liver collection $(N=1)$, bile leakage $(N=1)$ and cholecystitis $(N=2)$. Stent occlusion occurred in 4 patients $(10 \%)$ within a week as a result of tumour ingrowth $(N=3)$ or biliary sludge $(N=1)$. Two (5\%) stents occluded between 7 days and one month. Three (7.5\%) patients died during hospital admission owing to pre-existing cholangitic sepsis $(N=2)$ and pneumonia $(N=1)$. Six patients $(15 \%)$ survived less than one month, 10(25\%) survived between 1 and 3 months, 8 (20\%) survived between 3 and 6 months, and $9(22.5 \%)$ survived beyond 6 months. Seven $(17.5 \%)$ patients from distant referral institutes were not seen after discharge.

Conclusion. These data demonstrate that percutaneously placed SEMS achieve satisfactory palliation with a low complication rate in a highrisk patient group with advanced malignant biliary obstruction.

\section{Continuing medical education in Radiology in South Africa}

\author{
(continued from page 50)
}

governance and CME sponsorships/educational grants at both corporate and individual levels.

I am delighted to report that the RSSA CPD fund that was established in 1999 will formally be constituted as the official RSSA CME fund (an association not for gain) and will become the custodian of the RSSA CME in South Africa, Namibia and Botswana. The RSSA CME fund currently supports the RSSA travel award of R40 000 for the best paper by a registrar or junior radiologist, as well as the best poster prizes of R15 000 and R5 000 respectively. The R1 000 quiz case prize in the $S A J R$ is RSSA-sponsored, and various ad hoc allocations have been made. There are other initiatives, such as financial support for departmental libraries and increasing the capacity of the SAJR, under consideration. A major project under consideration is the establishment of a local RSSA visiting professor programme whereby local experts conduct courses and visit departments for short periods. The Current Ethical Issues courses are the prototype of such an initiative. I have a personal vision that the RSSA CME fund will in the medium term be strong enough to fund one or more RSSA chairs in radiology (the benefit to radiology of the privately sponsored chair of Professor Lotz at Stellenbosch is widely acknowledged), and this concept should be expanded to support other full-time faculty, fellowship or research posts.
During the presidential address, Professor McLoud ${ }^{1}$ reminded radiologists that they found themselves in a ' $24 / 7$ ' environment in imaging, and that we face new demands from both patients and referring clinicians. Global tele-medicine and tele-radiology are realities. They continue to grow as our profession evolves. The future of radiology hinges on the decisions we make today about how we educate and train in this new global environment. If we are to guarantee our specialty's future, we must be viewed as representing the highest level of specialty and sub-specialty care.

In conclusion: the new RSSA CME programme aims to instill a new co-operative attitude among all radiologists, academic institutions and industry stakeholders in Southern Africa, with reliable and prestigious international partners. Most importantly, the RSSA CME fund must be financially strong enough to keep the programme affordable, sustainable and enjoyable, with contented members, all contributing to maintain radiology as a vibrant, growing and relevant specialty.

\section{Leon Janse van Rensburg}

RSSA Congress Chair

1. McLoud TC. Trends in radiologic training: National and international implications. Radiology http://radiology.rsna.org/content/256/2/343.full (accessed 26 July 2010). 